\section{Corticosteroids and infliximab impair the performance of interferon- $\gamma$ release assays used for diagnosis of latent tuberculosis}

\begin{abstract}
The impact of immunosuppression on interferon- $\gamma$ release assays and novel cytokine biomarkers of TB infection, mycobacteriaspecific IL-2, IP-10 and TNF- $\alpha$ responses was investigated in an ex vivo model. Cytokine responses in standard QuantiFERON-TB Gold in-Tube (QFT-GIT) assays were compared with duplicate assays containing dexamethasone or infliximab. Dexamethasone converted QFT-GIT results from positive to negative in $30 \%$ of participants. Antigen-stimulated interferon- $\gamma$, IL-2 and TNF- $\alpha$ responses were markedly reduced, but IP-10 responses were preserved. Infliximab caused QFT-GIT result conversion in up to $30 \%$ of participants and substantial reductions in all cytokine responses. Therefore, corticosteroids and anti-TNF- $\alpha$ agents significantly impair interferon- $\gamma$ release assay performance. IP-10 may be a more robust TB biomarker than interferon- $\gamma$ in patients receiving corticosteroids.
\end{abstract}

\section{INTRODUCTION}

Drugs inhibiting TNF- $\alpha$, a key cytokine in autoimmune and antimicrobial inflammation, are increasingly used to treat chronic inflammatory conditions, including inflammatory bowel disease and rheumatoid arthritis. However, anti-TNF$\alpha$ agents greatly increase the risk of progression from latent TB infection (LTBI) to active $\mathrm{TB} .{ }^{1}$ Consequently, many guidelines recommend screening patients for LTBI prior to commencing anti-TNF- $\alpha$ therapy with interferon- $\gamma$ release assays (IGRAs). ${ }^{2}$ QuantiFERON-TB Gold is currently the most widely used IGRA in clinical practice. ${ }^{3}$

The key issue with using IGRAs in this setting is that the majority of patients are already receiving immunosuppressive drugs when anti-TNF- $\alpha$ therapy initiation is considered. ${ }^{4}$ IGRAs are functional assays based on interferon- $\gamma$ production by T cells in response to stimulation with TB-specific antigens in vitro. ${ }^{5}$ Reduction in $\mathrm{T}$ cell numbers (eg, in HIV infection) results in impaired IGRA performance. ${ }^{6}$ Thus, any functional impairment of T cells, including iatrogenic immunosuppression, will likely impact IGRA performance.

Several clinical studies have investigated IGRAs for LTBI diagnosis in patients who are immunosuppressed. ${ }^{7} 8$ However, all have one significant limitation-the lack of a gold standard to compare test performance against. In contrast to active $\mathrm{TB}$, where microbiological detection of Mycobacterium tuberculosis (Mtb) remains the gold standard, no such standard exists for LTBI. ${ }^{5}$ Therefore, interpretation of IGRAs in patients who are immunosuppressed is complex, as it is impossible to determine whether a negative result reflects absence of TB infection or alternatively a false-negative result caused by immunosuppressive agents.

A recent meta-analysis further supports immunosuppressive drugs interfering with IGRAs. ${ }^{9}$ Patients receiving corticosteroids or anti-TNF- $\alpha$ agents had significantly lower rates of positive IGRA results than those not treated with these agents. However, this meta-analysis had limitations, primarily related to insufficient data included in the original reports. Importantly, many lacked data on concomitant medication, and therefore this meta-analysis may have overestimated the effect of individual drugs.

We aimed to determine the impact of corticosteroids and infliximab on the performance of QuantiFERON-TB Gold In-Tube (QFT-GIT) assays using an ex vivo model. Additionally, the effect of these immunosuppressive agents on mycobacteria-specific IL-2, IP-10 and TNF- $\alpha$ responses, recently identified biomarkers of TB infection, was investigated. ${ }^{10} 11$

\section{METHODS}

Patients with a previous positive IGRA result or recent TB contact were recruited following informed consent. Potential participants with known immunodeficiency, receiving immunosuppressive medication, or with uncontrolled diabetes mellitus were excluded. From each participant, four QFT-GIT assay sets (Cellestis/Qiagen, Carnegie, Australia) comprising an antigen-stimulated, a positive control and a negative control sample were obtained. In the first set, no reagents were added ('standard assay'). In the second set, dexamethasone (Organon Laboratories, Cambridge, UK) was added to each tube at $2 \mu \mathrm{g} / \mathrm{ml}$, based on pharmacokinetic data. ${ }^{12}$ In the third and fourth sets, infliximab (MSD, Hoddesdon, UK) was added to each tube at $5 \mu \mathrm{g} / \mathrm{ml}$ ('low dose') and $100 \mu \mathrm{g} / \mathrm{ml}$ ('high dose'), respectively, corresponding to therapeutic trough and peak levels. ${ }^{13}$ Samples were then incubated within 4 hours of phlebotomy at $37^{\circ} \mathrm{C}$ for 24 hours, as per the manufacturer's instructions. Supernatants were then harvested and cryopreserved for batched analysis.

Cytokine concentrations were determined with ProcartaPlex xMAP assays (eBioscience, Hatfield, UK) measuring interferon- $\gamma$, IP-10, IL-2 and TNF- $\alpha$ following the manufacturer's instruction. Their broad dynamic range allows accurate measurement of high interferon- $\gamma$ concentrations that frequently occur in QFT-GIT samples, exceeding the upper limit of QFT-GIT ELISAs. ${ }^{14}$ Cytokine concentrations were measured using a Luminex 100 Bioanalyzer (Luminex, Austin, Texas, USA). QFT-GIT results were interpreted according to the manufacturer's package insert (latest UK version). ${ }^{15}$

Interferon- $\gamma$ concentrations measured in $\mathrm{pg} / \mathrm{mL}$ were converted to $\mathrm{IU} / \mathrm{mL}$ (the units used in QFT-GIT), as previously described. ${ }^{16}$ All other cytokines were analysed in $\mathrm{pg} / \mathrm{mL}$. Statistical analyses were performed in Prism (V5.0; GraphPad, La Jolla, California, USA). Wilcoxon matchedpairs signed-rank tests were used to compare cytokine concentrations in standard assays with those in assays with added drugs.

\section{RESULTS}

Nineteen adults (12 men; 7 women), median age 45 (range 20-68) years, were recruited. None of the participants had been started on anti-TB treatment prior to recruitment. HIV testing was performed in 16 participants (three declined testing); all had negative test results. Ten participants had positive QFT-GIT results in the standard assay, while nine were negative. Consequently, for analyses of antigenstimulated cytokine responses only data from the former 10 participants were included, while data from all 19 were included in analyses related to mitogen (positive control) responses.

\section{Interferon- $\gamma$ responses and categorical QFT-GIT results}

Of the 10 participants with positive QFT-GIT results in the standard assay, three had negative QFT-GIT results in the set with added dexamethasone, while seven remained positive. In the set with low-dose infliximab, two had negative QFT-GIT results, and eight remained positive. In the set with high-dose infliximab, two had negative QFT-GIT results and one had an indeterminate result; in seven the result remained positive.

Compared with the standard assay, the background-corrected interferon- $\gamma$ concentrations in antigen-stimulated samples were significantly lower with all added drugs (figure 1). Dexamethasone resulted in a 4.8-fold reduction; low-dose infliximab 
and high-dose infliximab resulted in 8.5fold and 16.4-fold reductions, respectively (table 1). In each participant, backgroundcorrected interferon- $\gamma$ concentrations in the antigen-stimulated samples were universally lower with added dexamethasone or infliximab (low- and high-dose) than in the standard assay (see online supplementary figure S1).

In the positive control samples, dexamethasone caused a significant reduction in the background-corrected interferon- $\gamma$ concentrations; in contrast, infliximab had no significant impact on positive control responses (figure 1).

\section{IL-2, IP-10 and TNF- $\alpha$ responses}

Dexamethasone resulted in reduced background-corrected IL-2 and TNF- $\alpha$ concentrations in antigen-stimulated samples (figure 1). Although the average reduction of both cytokines was substantial, this was only statistically significant for IL-2 (table 1). In contrast, dexamethasone had no substantial impact on background-corrected IP-10 concentrations in antigen-stimulated and positive control samples (figure 1 and table 1 ). Both low-dose and high-dose infliximab resulted in a significant reduction of background-corrected IL-2, IP-10 and TNF- $\alpha$ concentrations in antigenstimulated samples (figure 1 and table 1).

\section{DISCUSSION}

To our knowledge, this is the first study to use an ex vivo model to determine the impact of corticosteroids and infliximab on the performance of QFT-GIT assays. Importantly, this approach overcomes the key limitation of previous clinical studies that were hindered by the absence of a gold standard for LTBI.

We show that corticosteroids impair the performance of QFT-GIT assays significantly. On average, antigen-stimulated interferon- $\gamma$ responses were almost fivefold lower with dexamethasone compared with the standard assays. Our finding that dexamethasone at therapeutic concentrations led to QFT-GIT assay results changing from positive to negative in $30 \%$ of participants is consistent with our recent study that investigated the impact of antituberculous antibiotics and corticosteroids on QFT-GIT assays, where result conversion with dexamethasone occurred in 4 of 10 participants. ${ }^{17}$ The observation that dexamethasone also reduces positive control responses in QFT-GIT assays may explain the comparatively high proportions of indeterminate QFT-GIT results observed in patients receiving oral corticosteroids in previous clinical studies. ${ }^{18}$
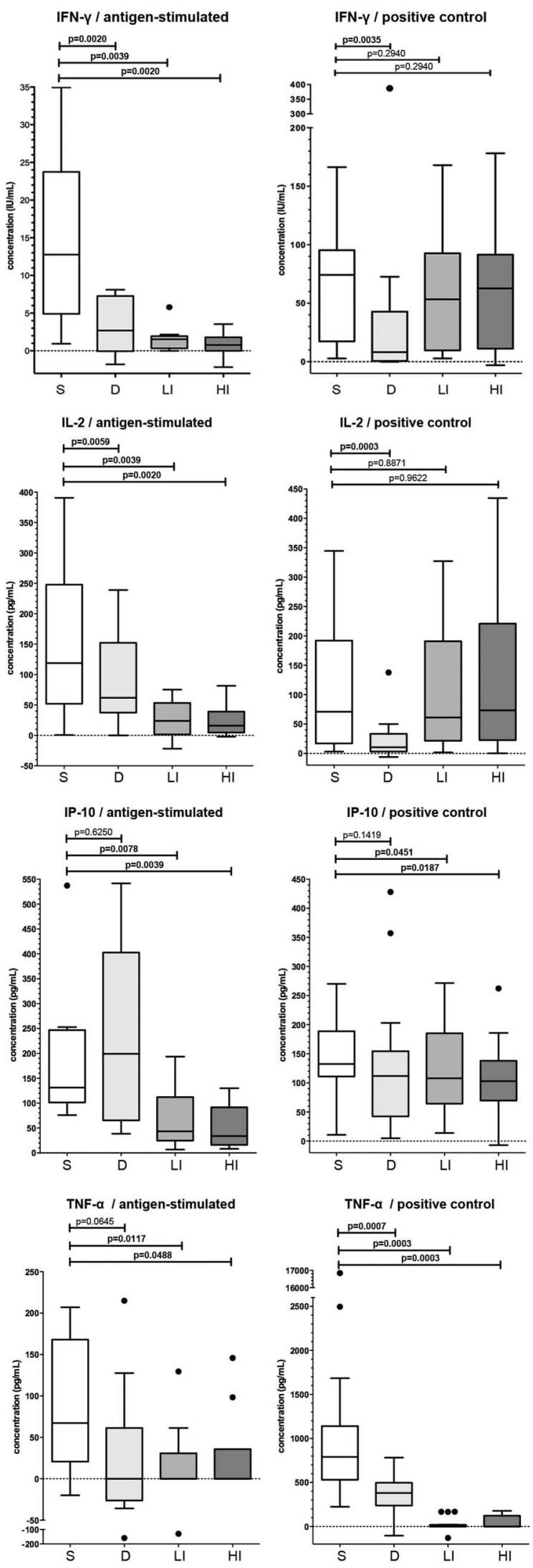

Figure 1 Antigen-stimulated and positive control interferon- $\gamma$, IL-2, IP-10 and TNF- $\alpha$ responses. Box plot with Tukey whiskers showing background-corrected cytokine concentrations in antigen-stimulated (left) and positive control (right) samples in standard (S) QFT-GIT assays (no additive) and those with added dexamethasone (D), low-dose infliximab (LI) and high-dose infliximab (HI). The horizontal lines depict the medians. The $p$ values were calculated with two-tailed Wilcoxon matched-pairs signed-rank tests. IFN- $\gamma$ : interferon- $\gamma ;$ QFT-GIT, QuantiFERON-TB Gold In-Tube. 
Table 1 Background-corrected cytokine concentrations in antigen-stimulated QFT-GIT samples

\begin{tabular}{llllllll}
\hline & $\begin{array}{l}\text { Standard assay } \\
\text { median conc. } \\
\text { (IQR)* }\end{array}$ & $\begin{array}{l}\text { Dexamethasone } \\
\text { median conc. } \\
(\text { IQR) }\end{array}$ & $\begin{array}{l}\text { Dexamethasone } \\
\text { ratiot }\end{array}$ & $\begin{array}{l}\text { Low-dose } \\
\text { iffliximab } \\
\text { median conc. } \\
\text { (IQR) }\end{array}$ & $\begin{array}{l}\text { Low-dose } \\
\text { infliximab } \\
\text { ratiot }\end{array}$ & $\begin{array}{l}\text { High-dose } \\
\text { infliximab } \\
\text { median conc. } \\
\text { (IQR)* }\end{array}$ & $\begin{array}{l}\text { High-dose } \\
\text { infliximab } \\
\text { ratiot }\end{array}$ \\
\hline IFN- $\gamma$ & $12.8(4.9-23.7)$ & $2.7(-0.1-7.3)$ & -4.8 & $1.5(0.33-1.9)$ & -8.5 & $0.8(-2.1-1.8)$ \\
IL-2 & $118.9(52.0-247.9)$ & $61.9(37.6-152.3)$ & -1.9 & $23.9(1.6-53.4)$ & -5.0 & $16.1(4.7-39.2)$ & -16.4 \\
IP-10 & $131.4(101.3-246.7)$ & $199.2(65.6-402.7)$ & +0.7 & $43.4(24.8-112.2)$ & -3.0 & $33.9(16.1-91.7)$ & -3.9 \\
TNF- $\alpha$ & $67.3(20.8-168.0)$ & $0.0(-26.2-61.3)$ & $-\ddagger$ & $0.0(0.0-30.8)$ & $-\ddagger$ & $0.0(0.0-145.8)$ & $-\ddagger$ \\
\hline
\end{tabular}

Only data from participants with a positive QFT-GIT result in the standard assay were included in this analysis $(n=10)$.

${ }^{*}$ Cytokine concentrations in $\mathrm{pg} / \mathrm{mL}$, except interferon- $\gamma$ concentrations $(\mathrm{IU} / \mathrm{mL})$.

tRatios were calculated by dividing median background-corrected cytokine concentrations in standard assays by median background-corrected cytokine concentrations in assays with added drugs.

¥Value cannot be calculated as one of the cells contains a zero value.

conc., concentration; IFN- $\gamma$ : interferon- $\gamma$; QFT-GIT, QuantiFERON-TB Gold In-Tube.

These findings are consistent with in vitro studies showing that corticosteroids inhibit pro-inflammatory cytokine production from T cells, including interferon- $\gamma .{ }^{19}$

Our observations are highly relevant to clinical practice, as they show that IGRAs may be unreliable in patients receiving corticosteroids. Therefore, the recommendation to screen patients on 'conventional' immunosuppressive therapy with IGRAs before starting biological therapy, as suggested by most guidelines, is flawed. Based on first principles it is probable that other immunosuppressive agents targeting $\mathrm{T}$ cell function, including calcineurin inhibitors and purine analogues, also impair IGRA performance. Consequently, guidelines should be amended so that IGRA testing is performed when a patient is first diagnosed with a disease that may ultimately require treatment with biological agents, rather than after initiation of immunosuppressive treatment. In patients already receiving immunosuppressive drugs, we and other experts recommend that IGRAs are used in conjunction with TSTs to increase diagnostic yield, as detailed elsewhere. $^{2} 20$ Furthermore, patients with a negative IGRA result at diagnosis and subsequent TB exposure, or at risk of exposure due to travel to or residence in a high TB prevalence, should be re-screened prior to starting biological agents.

Our results show that in the presence of corticosteroids IP-10 responses were sustained, indicating that IP-10 may be a more robust marker of $\mathrm{TB}$ infection in patients receiving corticosteroids. The underlying mechanism for this observation remains uncertain. However, in contrast to interferon- $\gamma$, IP-10 is mainly produced by monocytes, endothelial cells and fibroblasts, and there are data suggesting that corticosteroids have only limited impact on intracellular inflammatory signalling in monocytes. ${ }^{21}$ Several recent studies, including our own, highlight the potential of Mtb-specific IP-10 responses as novel biomarkers for the diagnosis of LTBI and active TB in immunocompetent patients. ${ }^{10} 11$ One small study in immunocompromised patients with active TB reported that Mtb-specific IP-10 responses did not differ significantly from those in patients with active TB without immunocompromise, further supporting the notion that IP-10 may be a better biomarker than interferon- $\gamma$ in individuals who are immunocompromised. ${ }^{11}$

Our data also suggest that the performance of IGRAs is significantly impaired by infliximab. We observed a considerable reduction in Mtb-specific interferon- $\gamma$ responses, and changes in categorical results in up to $30 \%$ of patients. These observations are concordant with current concepts of human anti-mycobacterial immune responses. TNF- $\alpha$, produced by antigen-presenting cells (APCs), plays a critical role in T cell/APC interactions by aiding granuloma formation and stimulating Mtb-specific $\mathrm{T}$ cells to produce interferon- $\gamma^{22}$ Interferon- $\gamma$ stimulates TNF- $\alpha$ production in APCs, thereby closing the feedback loop. Anti-TNF- $\alpha$ agents interrupt this interaction, thereby diminishing the ability to contain mycobacteria, and reducing the ability to generate adequate anti-mycobacterial responses in functional assays, such as IGRAs. The latter is further supported by the observation that infliximab markedly reduced all four Mtb-specific cytokine responses investigated.

In conclusion, we provide evidence that corticosteroids and infliximab significantly impair QFT-GIT performance. A substantial proportion of patients with LTBI receiving either drug are likely to have false-negative results if tested with this IGRA. Therefore, LTBI screening should be performed at the time of diagnosis, not after the initiation of immunosuppressive treatment. Mtb-specific IP-10 responses may be a more robust marker of LTBI in patients receiving corticosteroids than interferon- $\gamma$ responses, which form the basis of commercial IGRAs.

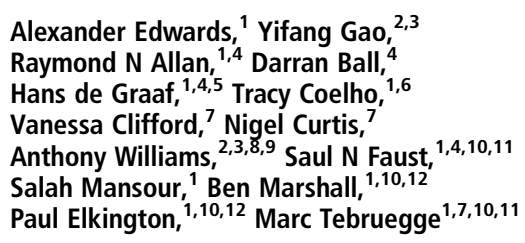

${ }^{1}$ Academic Unit of Clinical \& Experimental Sciences, Faculty of Medicine, University of Southampton, Southampton, UK

${ }^{2}$ Academic Unit of Cancer Sciences, Faculty of

Medicine, University of Southampton, Southampton, UK ${ }^{3}$ CRUK NIHR Southampton Experimental Cancer

Medicine Centre, University Hospital Southampton NHS Foundation Trust, Southampton, UK

${ }^{4} \mathrm{NIHR}$ Wellcome Trust Clinical Research Facility, University Hospital Southampton NHS Foundation Trust, Southampton, UK

${ }^{5}$ Department of Paediatric Rheumatology, University Hospital Southampton NHS Foundation Trust,

Southampton, UK

${ }^{6}$ Department of Paediatric Gastroenterology, University Hospital Southampton NHS Foundation Trust, Southampton, UK

${ }^{7}$ Department of Paediatrics, The University of Melbourne \& Murdoch Children's Research Institute, Parkville, Australia

${ }^{8}$ Department of Immunology, University Hospital Southampton NHS Foundation Trust, Southampton, UK ${ }^{9}$ Wessex Investigational Sciences Hub, University Hospital Southampton NHS Foundation Trust, Southampton, UK

${ }^{10}$ NIHR Respiratory Biomedical Research Unit, University Hospital Southampton NHS Foundation Trust, Southampton, UK

${ }^{11}$ Department of Paediatric Infectious Diseases \& Immunology, University Hospital Southampton NHS Foundation Trust, Southampton, UK

${ }^{12}$ Department of Respiratory Medicine, University Hospital NHS Foundation Trust, Southampton, UK

Correspondence to Dr Marc Tebruegge, NIHR Wellcome Trust Clinical Research Facility (Mailpoint 218), University Hospital Southampton NHS Foundation Trust, Tremona Road, Southampton S016 6YD, UK; m.tebruegge@soton.ac.uk

\section{Twitter Follow Marc Tebruegge @ResearchTB}

Contributors Study concept and design: $A E, Y G$, RNA, VC, NC, AW, PE, and MT; participant recruitment: $B M, P E$, and $M T$; sample processing and analysis: $A E$, $Y G, D B$, and $M T$; analysis of data: $A E, Y G, R N A, H d G$, 
TC, VC, NC, PE, and MT; data interpretation and drafting of the manuscript: $A E, V C, N C, S M, P E$, and MT. All authors critically read, commented on, and approved the final version of the manuscript.

Funding $H d G, D B, R N A$ and SNF are supported by the Southampton NIHR Wellcome Trust Clinical Research Facility; MT was supported by a Clinical Lectureship provided by the UK National Institute for Health Research.

Competing interests MT has received QuantiFERON-TB Gold assays at reduced cost for related research projects from the manufacturer (Cellestis/Qiagen). The manufacturer had no influence on the study design, data interpretation, writing of the manuscript or decision to submit the data for publication.

Ethics approval The study was approved by the National Research Ethics Service Committee South Central (approval number 13/SC/0043).

Provenance and peer review Not commissioned; externally peer reviewed.

- Additional material is published online only. To view please visit the journal online (http://dx.doi.org/10. 1136/thoraxjn-2016-209397).

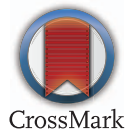

To cite Edwards A, Gao Y, Allan RN, et al. Thorax 2017;72:946-949.

Received 7 September 2016

Revised 19 December 2016

Accepted 12 January 2017

Published Online First 3 February 2017

Thorax 2017;72:946-949.

doi:10.1136/thoraxjnl-2016-209397

\section{REFERENCES}

1 Keane J, Gershon S, Wise RP, et al. Tuberculosis associated with infliximab, a tumor necrosis factor alpha-neutralizing agent. $N$ Engl I Med 2001;345:1098-104.
2 Hewitt RJ, Francis M, Singanayagam A, et al. Screening tests for tuberculosis before starting biological therapy. BMJ 2015;350:h1060.

3 Tebruegge M, Ritz N, Koetz K, et al. Availability and use of molecular microbiological and immunological tests for the diagnosis of tuberculosis in Europe. PLOS ONE 2014;9:e99129.

4 Hyrich KL, Watson KD, Lunt M, et al. Changes in disease characteristics and response rates among patients in the United Kingdom starting anti-tumour necrosis factor therapy for rheumatoid arthritis between 2001 and 2008. Rheumatology 2011;50:117-23.

5 Tebruegge M, Ritz N, Curtis N, et al. Diagnostic tests for childhood tuberculosis: past imperfect, present tense and future perfect? Pediatr Infect Dis J 2015;34:1014-19.

6 Cattamanchi A, Smith R, Steingart KR, et al. Interferon-gamma release assays for the diagnosis of latent tuberculosis infection in HIV-infected individuals: a systematic review and meta-analysis. J Acquir Immune Defic Syndr 2011;56:230-8.

7 Casas S, Andreu A, Juanola X, et al. Diagnosis of tuberculosis infection by tuberculin skin test and a whole-blood interferon- $\gamma$ release assay in patients considered for anti-tumor necrosis factor- $\alpha$ therapy. Diagn Microbiol Infect Dis 2011;71:57-65.

8 Greveson K, Goodhand J, Capocci S, et al. Yield and cost effectiveness of mycobacterial infection detection using a simple IGRA-based protocol in UK subjects with inflammatory bowel disease suitable for anti-TNF $\alpha$ therapy. J Crohns Colitis 2013:7:412-18

9 Wong SH, Gao Q, Tsoi KK, et al. Effect of immunosuppressive therapy on interferon $\gamma$ release assay for latent tuberculosis screening in patients with autoimmune diseases: a systematic review and meta-analysis. Thorax 2016;71:64-72.

10 Tebruegge M, Dutta B, Donath $\mathrm{S}$, et al. Mycobacteria-specific cytokine responses detect tuberculosis infection and distinguish latent from active tuberculosis. Am J Respir Crit Care Med 2015;192:485-99.

11 Ruhwald M, Bodmer T, Maier C, et al. Evaluating the potential of IP-10 and MCP-2 as biomarkers for the diagnosis of tuberculosis. Eur Respir $J$ 2008;32:1607-15.
12 Weijtens O, Schoemaker RC, Cohen AF, et al. Dexamethasone concentration in vitreous and serum after oral administration. Am J Ophthalmol 1998:125:673-9.

13 St Clair EW, Wagner CL, Fasanmade AA, et al. The relationship of serum infliximab concentrations to clinical improvement in rheumatoid arthritis: results from ATTRACT, a multicenter, randomized, double-blind, placebo-controlled trial. Arthritis Rheum 2002;46:1451-9.

14 Jarvis J, Gao Y, de Graaf $\mathrm{H}$, et al. Environmental temperature impacts on the performance of QuantiFERON-TB Gold In-Tube assays. J Infect 2015;71:276-80.

15 Cellestis/Qiagen. QuantiFERON-TB Gold Package Insert (Doc. No. US05990301L, Version March 2013). http://www.quantiferon.com/irm/content/PI/ QFT/2PK/US.pdf (accessed 8 Dec 2016)

16 Desem N, Jones SL. Development of a human gamma interferon enzyme immunoassay and comparison with tuberculin skin testing for detection of Mycobacterium tuberculosis infection. Clin Diagn Lab Immunol 1998;5:531-6.

17 Clifford V, Zufferey C, Germano S, et al. The impact of anti-tuberculous antibiotics and corticosteroids on cytokine production in QuantiFERON-TB Gold In Tube assays. Tuberculosis (Edinb) 2015;95:343-9.

18 Bélard E, Semb S, Ruhwald M, et al. Prednisolone treatment affects the performance of the QuantiFERON gold in-tube test and the tuberculin skin test in patients with autoimmune disorders screened for latent tuberculosis infection. Inflamm Bowel Dis 2011:17:2340-9.

19 Brinkmann V, Kristofic C. Regulation by corticosteroids of Th1 and Th2 cytokine production in human CD4+ effector T cells generated from CD45RO- and CD45RO+ subsets. J Immunol 1995; 155:3322-8.

20 Clifford V, Tebruegge M, Curtis N. Limitations of current tuberculosis screening tests in immunosuppressed patients. BMJ 2015;350:h2226.

21 Hu X, Li WP, Meng C, et al. Inhibition of IFN-gamma signaling by glucocorticoids. J Immunol 2003:170:4833-9.

22 van Crevel R, Ottenhoff TH, van der Meer JW. Innate immunity to Mycobacterium tuberculosis. Clin Microbiol Rev 2002;15:294-309. 